\title{
Analytical 1D transfer functions for layered soils
}

\author{
Joaquin Garcia-Suarez ${ }^{a}$ \\ Domniki Asimaki ${ }^{b}$ \\ ${ }^{a}$ Civil Engineering Institute, École Polytechnique Fédérale de \\ Lausanne (EPFL), Lausanne, Switzerland \\ ${ }^{b}$ Mechanical and Civil Engineering, Caltech, Pasadena, CA, U.S.A
}

\begin{abstract}
Transfer functions are constantly used in both Seismology and Geotechnical Earthquake Engineering to relate seismic displacement at different depths within strata. In the context of Diffusive Theory, they also appear in the expression of the imaginary part of 1D Green's functions. In spite of its remarkable importance, their mathematical structure is not fully understood yet, except in the simplest cases of two or three layers at most. This incomplete understanding, in particular as to the effect of increasing number of layers, hinders progress in some areas, as researchers have to resort to expensive and less conclusive numerical parametric studies. This text presents the general form of transfer functions for any number of layers, overcoming the above issues. Owing to the formal connection between seismic wave propagation and other phenomena that, in essence, represent different instances of wave propagation in a linear-elastic medium, one can extend the results derived elsewhere [Garcia-Suarez, Joaquin. 2021. "Trace Spectrum of $1 D$ Transfer Matrices for Wave Propagation in Layered Media." engrXiv. June 24. doi:10.31224/osf.io/ygt8z.] in the context of longitudinal wave propagation in modular rods to seismic response of stratified sites. The knowledge of the general closed-form expression of the transfer functions allows to analytically characterize the long-wavelength asymptotics of the horizontal-to-vertical spectral ratio for any number of layers.
\end{abstract}

\section{Introduction}

The study of elastic-wave propagation phenomena is of utmost interest to both the Seismology and the Geotechnical Earthquake Engineering communities: the sudden release of energy created by a fault rupturing generates a deformation field that propagates towards the free surface and it is ultimately felt as an earthquake. This wavefield is usually studied using the tools of Linear Elasticity 


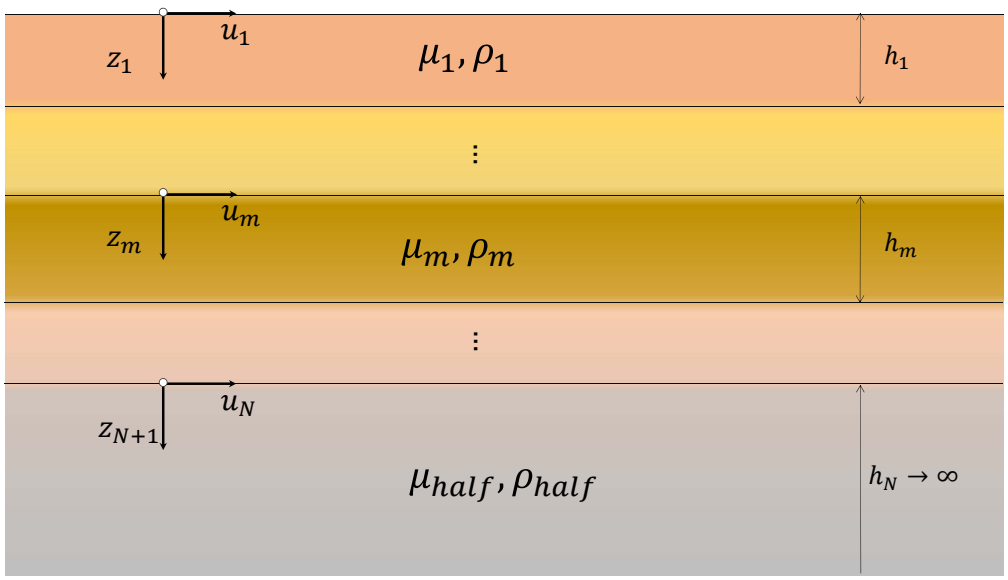

Figure 1: Scheme of the layered system and local coordinates.

[1], except when the propagation takes place within soft soils, what requires considering material non-linear response [2]. It is also the case that when the front arrives to the shallow layers, assuming the earthquake source is located deep enough, it does so almost-vertically (the decreasing stiffness bends the rays towards the normal of the free surface). Moreover, for the length-scales of interest in applications, the local curvature of the wavefield is relatively small compared to the window of observation, so the impinging excitation can be considered to be made up of plane waves.

Under the assumption of one-dimensional propagation perpendicular to the free surface, the different components decouple and SH, SV and P waves can be studied separately and then summed up, as there is no mode conversion.

A customary method to study the evolution of the wave fields across the layers is the so-called "Propagator Matrix Method", also referred as "Method of Matrizants" in older texts [3]. An equivalent matrix approach for the 1D case is presented in [2], and used often in geotechnical engineering. The classic Seismology texts $[1,4]$ present a general treatment for inclined $\mathrm{SH}$ waves and inclined P-SV waves; however, in this work, we will consider no inclination, thus the expressions that appear in the prior references can be simplified.

These approaches based on propagator matrices assign a matrix to every layer in the site, which depends on the mechanical properties of the material the layer is made of as well as on the ratio between the wavelength of the propagating plane wave to the thickness of the layer. These matrices link stresses and displacements in one border of the layer to the other one. Among other usages, this method allows obtaining "transfer functions" to relate the displacement amplitude at the free surface to either the displacement at a deeper stratum or to the amplitude of the wave coming from an idealized half-space that impinges on the shallow layers.

Even though these transfer functions are usually evaluated numerically, there 
is still room for closed-form analysis. The general expression for transfer functions in sites with any number of layers is presented and then verified against numerical evaluation following the usual numerical matrix-multiplication procedure. Finally, a relevant application of the new closed-form formulae is explored: obtaining analytical expression for the ratio between horizontal and vertical spectral surface amplitude components in the long-wavelength limit, following the pioneer of Kawase and colleagues [5].

Before concluding this introduction, let us mention that the elastic wave propagation in layered media is of interest to the Solid Mechanics community at large, in particular, also to researchers interested in dispersion relations in periodic structures [6,7]. It is in this context that the general form of the N-layer propagator has recently been introduced, see [8]. The mathematical problem is the same in both cases, just its guise is changed to the seismological one we are considering in this text. Since the derivation of the general form of the entries of the global propagator is presented in [8], it will not be included in the text and the reader interested in such details is referred to that publication. Beyond Elasticity, electromagnetic waves [9] and acoustic waves [10] propagating in layered media in unidirectional fashion also adopt a similar mathematical form, among others.

\section{Closed-form expression of general transfer func- tions}

\subsection{Brief refresh of the mathematical modeling}

Let us illustrate the method using $\mathrm{S}$ waves at first, yet considering $\mathrm{P}$ waves instead only requires swapping the medium shear modulus with the corresponding bulk modulus. We assume that the Fourier transform has been applied to the equations of the problem, thus the results are given in the frequency domain. The method considers balance of linear momentum (equilibrium) and the constitutive relation (relation stress-strain). Under the 1D assumption, frequencydomain, for $\mathrm{S}$ waves, these equations in matrix form read as:

$$
\frac{d \boldsymbol{f}}{d z}=\frac{d}{d z}\left[\begin{array}{c}
\hat{u} \\
\hat{\tau}_{x z}
\end{array}\right]=\left[\begin{array}{cc}
0 & 1 / \mu_{k} \\
-\rho_{k} \omega^{2} & 0
\end{array}\right]\left[\begin{array}{c}
\hat{u} \\
\hat{\tau}_{x z}
\end{array}\right]=\boldsymbol{A}_{k} \boldsymbol{f}(z),
$$

where $z$ is the vertical coordinate, see Figure 1, $\hat{u}$ represents the Fourieramplitude (frequency-domain amplitude) of the horizontal displacement, $\hat{\tau}_{x z}$ is the amplitude of the shear stresses, $\mu_{k}$ and $\rho_{k}$ refer respectively to the shear modulus and the density of the soil, and $\omega$ is the circular frequency of the propagating wave. Later on, we will also discuss the effect of hysteretic damping in the soil, hence we will use the symbol $\delta_{d, k}$ to refer to the damping coefficient of the $k$-th layer (recall that, invoking the Equivalence Principle [4], introducing this kind of dissipation mechanism in the frequency-domain response of the material amounts to substituting the real shear modulus $\mu_{k}$ by a complex one given by $\left.\mu_{k}\left(1+\mathrm{i} \delta_{d, k}\right)\right)$. 
We assume that conditions at the surface of the site are given by $\boldsymbol{f}(z=$ $0)=\left[\hat{u}_{t o p}, 0\right]^{\top}$, where $\hat{u}_{\text {top }}$ is the unknown ground displacement and the stress is zero as there are no forces imposed on this contour. The vector $\boldsymbol{f}$ is called the motion-stress vector, the matrix $\boldsymbol{A}_{k}$ is referred to as the $k$-th layer matrix. For a given frequency, the matrix layer is a constant within the layer as mechanical properties remain constant themselves.

\subsection{Transfer functions}

To conclude with the review, we remind the reader of the two kinds of transfer functions that appear profusely in the literature.

\subsubsection{Displacement-displacement}

The stress-free surface translates into a zero entry of the motion-stress vector at $z=z_{0}=0$ :

$$
\boldsymbol{f}(0)=\left[\begin{array}{ll}
\hat{u}_{\text {top }} & 0
\end{array}\right]^{\top}
$$

This means that the relation between the displacement at the free-surface, $\hat{u}_{t o p}$ and the displacement at any other interface, say, the k-th one, comes given by

$$
\boldsymbol{f}\left(z=z_{k}\right)=\left[\begin{array}{c}
\hat{u} \\
\hat{\tau}_{x z}
\end{array}\right]_{z=z_{k}}=\boldsymbol{P}\left(z_{k}, z_{0}\right)\left[\begin{array}{c}
\hat{u}_{t o p} \\
0
\end{array}\right] \rightarrow T F_{u-u}(\omega)=\frac{\hat{u}_{t o p}}{\hat{u}_{b}}=\frac{1}{P_{11}\left(z_{k}, z_{0}\right)}
$$

the displacement at the base (interface with the half-space) is $\hat{u}_{b}=\hat{u}\left(z=z_{k}\right)$

\subsubsection{Displacement-impinging amplitude}

Let us focus on the last layer overlying the half-space. This expression has also been considered in the past $[11,5]$. The displacement at the base of the last layer is equal to the one at the top of the half-space, which in turn is given by the superposition of the impinging wave and the transmitted back into the half-space after traversing the upper layered soil back and forth. Following the traditional seismological notation, $\dot{S}$ represents the upward-going wave amplitude, while $\grave{S}$, the down-going one. Thus, we can write the base-to-top relation as

$\boldsymbol{f}\left(z=z_{k}\right)=\left[\begin{array}{c}\hat{u} \\ \hat{\tau}_{x z}\end{array}\right]_{z=z_{k}}=\left[\begin{array}{c}\grave{S}+\grave{S} \\ \mu_{\text {half }} \operatorname{ik}(\grave{S}-\dot{S})\end{array}\right]_{z=z_{k}}=\boldsymbol{P}\left(z_{k}, z_{0}\right)\left[\begin{array}{c}\hat{u}_{\text {top }} \\ 0\end{array}\right]=\left[\begin{array}{l}P_{11} \hat{u}_{t o p} \\ P_{21} \hat{u}_{\text {top }}\end{array}\right]$,

thus the ratio $\hat{u}_{t o p} / \dot{S}$ can be written as

$$
\mathrm{TF}(\omega)=\frac{\hat{u}_{t o p}}{\dot{S}}=\frac{2}{P_{11}+\mathrm{i} \frac{P_{21}}{\omega \sqrt{\rho_{h a l f} \mu_{h a l f}}}} .
$$


Let us state the obvious relation between the two transfer functions:

$$
\frac{2}{\mathrm{TF}}=\frac{1}{T F_{u-u}}+\mathrm{i} \frac{P_{21}}{\omega \sqrt{\rho_{\text {half }} \mu_{\text {half }}}} .
$$

Notice that the subscript " $u-u$ " and italic font are being used to distinguish one transfer function from the other. Later in the text, the subscript " $p$ " and " $s$ " will be attached to TF to clarify if its refer to either pressure or shear wave propagation.

\subsection{Displacement-to-displacement transfer function gen- eral expression}

As mentioned above, the detailed derivation of the general form of the global propagator can be consulted in [8]. Since the upcoming results make extensive use of multi-indices, an appendix with a review on the subject is added at the end of the document.

The general term for the displacement-to-displacement transfer function, in a site with $N$ layers is

$$
T F_{u-u}(\omega)^{-1}=\left(\prod_{i=1}^{N} \cos \left(r_{i}\right)\right)\left(\sum_{\beta=0}^{\lfloor N / 2\rfloor} \sum_{|\mathbf{b}|=2 \beta} C_{\mathrm{b}} \tan (r)^{\mathrm{b}}\right),
$$

where $\lfloor\cdot\rfloor$ is the floor function, $r_{i}=\omega h_{i} / \sqrt{\mu_{i} / \rho_{i}}$, the coefficients $C_{\mathrm{b}}$ being given by

$$
C_{\mathrm{b}}=(-1)^{|\mathrm{b}| / 2}(\sqrt{\rho \mu})^{\mathrm{f}(\mathbf{b})},
$$

each binary multi-index $\mathrm{b} \in(\{0,1\})^{N}$ codifies the effect of the impedance contrast at the interfaces, and the map $\mathrm{f}:(\{0,1\})^{N} \rightarrow(\{-1,0,1\})^{N}$ takes the multi-index $b$, entry-wise, to the multi-index $\mathrm{f}(\mathrm{b})$ defined as:

- if $\mathrm{b}_{i}=0$, then $\mathrm{f}\left(\mathrm{b}_{i}\right)=0$,

- if $\mathrm{b}_{i}=1$ and the previous value assigned by $\mathrm{f}$ to the prior 1-entry in $\mathrm{b}$ was -1 , then $\mathrm{f}\left(\mathrm{b}_{i}\right)=1$, else $\mathrm{f}\left(\mathrm{b}_{i}\right)=-1$.

The total number of addends in the sum (each addend being a product of certain number of tangents except for the first one that is simply 1) is equal to

$$
N_{\text {addends }}=1+\left(\begin{array}{c}
N \\
2
\end{array}\right)+\left(\begin{array}{c}
N \\
4
\end{array}\right)+\ldots+\left(\begin{array}{c}
N \\
\lfloor N / 2\rfloor
\end{array}\right)=\sum_{k=1}^{\frac{1}{2}\lfloor N / 2\rfloor}\left(\begin{array}{c}
N \\
2 k
\end{array}\right)=2^{N-1}
$$

\subsubsection{Example: $N=2$}

Let us detail how the multi-index notation unfolds to yield the well-known result [12] for the simplest possible size. Since $N=2$, there can only be two multiindices of even degree:

$$
\mathrm{b}=(0,0) \rightarrow \mathrm{f}(\mathrm{b})=(0,0) \rightarrow C_{(0,0)}=1,
$$




$$
\mathrm{b}=(1,1) \rightarrow \mathrm{f}(\mathrm{b})=(1,-1) \rightarrow C_{(1,1)}=\frac{\sqrt{\rho_{1} \mu_{1}}}{\sqrt{\rho_{2} \mu_{2}}} .
$$

Thus we obtain the case studied by Madera [12]:

$$
T F_{u-u}(\omega)^{-1}=\cos \left(r_{1}\right) \cos \left(r_{2}\right)\left(1-\sqrt{\frac{\rho_{1} \mu_{1}}{\rho_{2} \mu_{2}}} \tan \left(r_{1}\right) \tan \left(r_{2}\right)\right),
$$

see also that the second term between parenthesis when equalized to zero provides an equation for the natural frequencies of the system, for this case and for every other number of layers greater than two.

\subsubsection{Example: $N=3$}

For three layers: we have the following possible $b$ values, which are mapped by $\mathrm{f}$ as

$$
\begin{aligned}
& \mathrm{b}=(0,0,0) \rightarrow \mathrm{f}(\mathrm{b})=(0,0,0) \rightarrow(\sqrt{\rho \mu})^{\mathrm{b}}=1, \\
& \mathrm{~b}=(1,1,0) \rightarrow \mathrm{f}(\mathrm{b})=(1,-1,0) \rightarrow(\sqrt{\rho \mu})^{\mathrm{b}}=\frac{\sqrt{\rho_{1} a_{1}}}{\sqrt{\rho_{2} a_{2}}}, \\
& \mathrm{~b}=(1,0,1) \rightarrow \mathrm{f}(\mathrm{b})=(1,0,-1) . \rightarrow(\sqrt{\rho \mu})^{\mathrm{b}}=\frac{\sqrt{\rho_{1} a_{1}}}{\sqrt{\rho_{3} a_{3}}} \\
& \mathrm{~b}=(0,1,1) \rightarrow \mathrm{f}(\mathrm{b})=(0,1,-1) \rightarrow(\sqrt{\rho \mu})^{\mathrm{b}}=\frac{\sqrt{\rho_{2} a_{2}}}{\sqrt{\rho_{2} a_{2}}}
\end{aligned}
$$

thus, from eq. (7),

$$
\begin{aligned}
T F_{u-u}(\omega)^{-1}=\cos \left(r_{1}\right) \cos \left(r_{2}\right) \cos \left(r_{3}\right)(1 & -\sqrt{\frac{\rho_{1} \mu_{1}}{\rho_{2} \mu_{2}}} \tan \left(r_{1}\right) \tan \left(r_{2}\right) \\
& -\sqrt{\frac{\rho_{1} \mu_{1}}{\rho_{3} \mu_{3}}} \tan \left(r_{1}\right) \tan \left(r_{3}\right) \\
& \left.-\sqrt{\frac{\rho_{2} \mu_{2}}{\rho_{3} \mu_{3}}} \tan \left(r_{2}\right) \tan \left(r_{3}\right)\right) .
\end{aligned}
$$

\subsection{Displacement-to-impinging-amplitude transfer function analytical expression}

Likewise, for $P_{21}$ in eq. (6) one has

$$
\omega \sqrt{\rho_{\text {half }} \mu_{\text {half }}} P_{21}=\left(\prod_{i=1}^{N} \cos \left(r_{i}\right)\right)\left(\sum_{\beta=0}^{\left\lfloor\frac{N-1}{2}\right\rfloor} \sum_{|\mathbf{b}|=1+2 \beta} S_{\mathrm{b}} \tan (r)^{\mathbf{b}}\right),
$$

the coefficients $S_{\mathrm{b}}$ being given by

$$
S_{\mathrm{b}}=(-1)^{(|\mathrm{b}|+1) / 2}(\sqrt{\rho \mu})^{\mathrm{g}(\mathrm{b})},
$$


where $g(b)$ has the same structure as $f(b)$ but acts from right to left instead of from left to right; since in this case the multi-index will always have degree odd, there will be an impedance that is left "hanging" (it will not enter into any impedance ratio), but it is divided by $\sqrt{\rho_{\text {half }} \mu_{\text {half }}}$ in the final expression.

In this case, the number of addends, one can verify, is also $2^{N-1}$. Combining eq. (14) and eq. (7) into eq. (6) yields the expression of the second transfer function:

$$
\begin{aligned}
2(\mathrm{TF})^{-1}=\left(\prod_{i=1}^{N} \cos \left(r_{i}\right)\right)\left(\sum_{\beta=0}^{\lfloor N / 2\rfloor} \sum_{|\mathrm{b}|=2 \beta} C_{\mathrm{b}} \tan (r)^{\mathrm{b}}\right. & \\
& \left.+\mathrm{i} \sum_{\beta=0}^{\left\lfloor\frac{N-1}{2}\right\rfloor} \sum_{|\mathrm{b}|=1+2 \beta} \frac{S_{\mathrm{b}}}{\sqrt{\rho_{\text {half }} \mu_{\text {half }}}} \tan (r)^{\mathrm{b}}\right)
\end{aligned}
$$

\subsubsection{Example: $\mathrm{N}=2$}

For two layers, there can only be two multi-indices of odd degree:

$$
\begin{aligned}
& \mathrm{b}=(1,0) \rightarrow \mathrm{g}(\mathrm{b})=(1,0) \rightarrow(-1)^{1} \tan \left(r_{1}\right)^{1} \tan \left(r_{2}\right)^{0} S_{(1,0)}=-\sqrt{\rho_{1} \mu_{1}} \tan \left(r_{1}\right), \\
& \mathrm{b}=(0,1) \rightarrow \mathrm{g}(\mathrm{b})=(0,1) \rightarrow(-1)^{1} \tan \left(r_{1}\right)^{0} \tan \left(r_{2}\right)^{1} S_{(0,1)}=-\sqrt{\rho_{2} \mu_{2}} \tan \left(r_{2}\right),
\end{aligned}
$$

thus, adding these terms to those obtained in eq. (11)

$$
\begin{aligned}
& 2(\mathrm{TF}(\omega))^{-1}=\cos \left(r_{1}\right) \cos \left(r_{2}\right)\left(1-\sqrt{\frac{\rho_{1} \mu_{1}}{\rho_{2} \mu_{2}}} \tan \left(r_{1}\right) \tan \left(r_{2}\right)\right. \\
& -\mathrm{i} \sqrt{\frac{\rho_{1} \mu_{1}}{\rho_{\text {half }} \mu_{\text {half }}}} \tan \left(r_{1}\right) \\
& \left.-\mathrm{i} \sqrt{\frac{\rho_{2} \mu_{2}}{\rho_{\text {half }} \mu_{\text {half }}}} \tan \left(r_{2}\right)\right) \text {. }
\end{aligned}
$$

Unlike the displacement-displacement transfer function, which only depends on the properties of the upper layers, in this expression the properties of the half-space do appear explicitly.

\subsubsection{Example: $\mathrm{N}=3$}

Likewise,

$$
\begin{aligned}
& \mathrm{b}=(1,0,0) \rightarrow \mathrm{g}(\mathrm{b})=(1,0,0) \rightarrow-\sqrt{\rho_{1} \mu_{1}} \tan \left(r_{1}\right) \\
& \mathrm{b}=(0,1,0) \rightarrow \mathrm{g}(\mathrm{b})=(0,1,0) \rightarrow-\sqrt{\rho_{1} \mu_{1}} \tan \left(r_{1}\right) \\
& \mathrm{b}=(0,0,1) \rightarrow \mathrm{g}(\mathrm{b})=(0,0,1) \rightarrow-\sqrt{\rho_{3} \mu_{3}} \tan \left(r_{3}\right) \\
& \mathrm{b}=(1,1,1) \rightarrow \mathrm{g}(\mathrm{b})=(1,-1,1) \rightarrow+\sqrt{\rho_{3} \mu_{3}} \frac{\sqrt{\rho_{1} \mu_{1}}}{\sqrt{\rho_{2} \mu_{2}}} \tan \left(r_{1}\right) \tan \left(r_{2}\right) \tan \left(r_{3}\right)
\end{aligned}
$$


so adding these terms to eq. (13)

$$
\begin{aligned}
T F_{u-u}(\omega)^{-1}=\cos \left(r_{1}\right) \cos \left(r_{2}\right) \cos \left(r_{3}\right)(1 & -\sqrt{\frac{\rho_{1} \mu_{1}}{\rho_{2} \mu_{2}}} \tan \left(r_{1}\right) \tan \left(r_{2}\right) \\
& -\sqrt{\frac{\rho_{1} \mu_{1}}{\rho_{3} \mu_{3}}} \tan \left(r_{1}\right) \tan \left(r_{3}\right) \\
& -\sqrt{\frac{\rho_{2} \mu_{2}}{\rho_{3} \mu_{3}}} \tan \left(r_{2}\right) \tan \left(r_{3}\right) \\
& -\mathrm{i} \sqrt{\frac{\rho_{1} \mu_{1}}{\rho_{\text {half }} \mu_{\text {half }}}} \tan \left(r_{1}\right) \\
& -\mathrm{i} \sqrt{\frac{\rho_{2} \mu_{2}}{\rho_{\text {half }} \mu_{\text {half }}}} \tan \left(r_{2}\right) \\
& -\mathrm{i} \sqrt{\frac{\rho_{3} \mu_{3}}{\rho_{\text {half }} \mu_{\text {half }}}} \tan \left(r_{3}\right) \\
& \left.+\mathrm{i} \sqrt{\frac{\rho_{3} \mu_{3}}{\rho_{\text {half }} \mu_{\text {half }}}} \sqrt{\frac{\rho_{1} \mu_{1}}{\rho_{2} \mu_{2}}} \tan \left(r_{1}\right) \tan \left(r_{2}\right) \tan \left(r_{3}\right)\right) .
\end{aligned}
$$

\section{Verification}

In order to verify the results we resort to computing numerical transfer functions (using both the conventional propagator method as outlined in Aki \& Richards' "Quantitative Seismology" [1] and the method outlined in Kramer's geotechnical engineering book [2]) and comparing them to the direct evaluation of the formulas in eq. (3) and eq. (4).

A list of ten Kik-net site layerings [13] has been used, the sites being those also studied in [14]. Only two examples are shown but the other eight are provided in an appendix. The information about these sites, code called "TKCH08" and "IWTH08", is provided in Tables 1 and 2.

The results display perfect agreement for both kinds of transfer functions (the numerical evaluation procedure is consigned into a Mathematica notebook [15] that is provided to the readers, see Supplementary Material section at the end of this article). Moreover, they allow to better understand the different shape of each kind of function: the "u-u" transfer functions do not contain odd powers of the tangents, see eq. (7), while the "u-S" transfer function does, eq. (16); the latter also contains twice as many terms as the former, hence its greater shape complexity and unintuitive evolution (compare Figure 5 to Figure 4 and Figure 3 to Figure 2) should not come as a surprise. 


\begin{tabular}{cccccc} 
Layer \# & $\begin{array}{c}\text { Thickness } \\
{[\mathrm{m}]}\end{array}$ & $\begin{array}{c}\text { Depth } \\
{[\mathrm{m}]}\end{array}$ & $\begin{array}{c}\text { S velocity } \\
{[\mathrm{m} / \mathrm{s}]}\end{array}$ & $\begin{array}{c}\text { P velocity } \\
{[\mathrm{m} / \mathrm{s}]}\end{array}$ & Poisson's ratio \\
\hline 1 & 4.0 & 4.0 & 150.0 & 360.0 & 0.39 \\
2 & 6.0 & 10.0 & 280.0 & 600.0 & 0.36 \\
3 & 10.0 & 20.0 & 280.0 & 2150.0 & 0.49 \\
4 & 14.0 & 34.0 & 680.0 & 3000.0 & 0.47 \\
5 & 16.0 & 50.0 & 900.0 & 3000.0 & 0.45 \\
6 & $\infty$ & $\infty$ & 2120.0 & 3680.0 & 0.45 \\
\hline
\end{tabular}

Table 1: Kik-net information site IWTH08.

\begin{tabular}{cccccc} 
Layer \# & $\begin{array}{c}\text { Thickness } \\
{[\mathrm{m}]}\end{array}$ & $\begin{array}{c}\text { Depth } \\
{[\mathrm{m}]}\end{array}$ & $\begin{array}{c}\text { S velocity } \\
{[\mathrm{m} / \mathrm{s}]}\end{array}$ & $\begin{array}{c}\text { P velocity } \\
{[\mathrm{m} / \mathrm{s}]}\end{array}$ & Poisson's ratio \\
\hline 1 & 4.0 & 4.0 & 130.0 & 300.0 & 0.38 \\
2 & 32.0 & 36.0 & 480.0 & 1850.0 & 0.46 \\
3 & 42.0 & 78.0 & 590.0 & 1850.0 & 0.44 \\
4 & $\infty$ & $\infty$ & 2800.0 & 5000.0 & 0.44 \\
\hline
\end{tabular}

Table 2: Kik-net information site TKCH08.
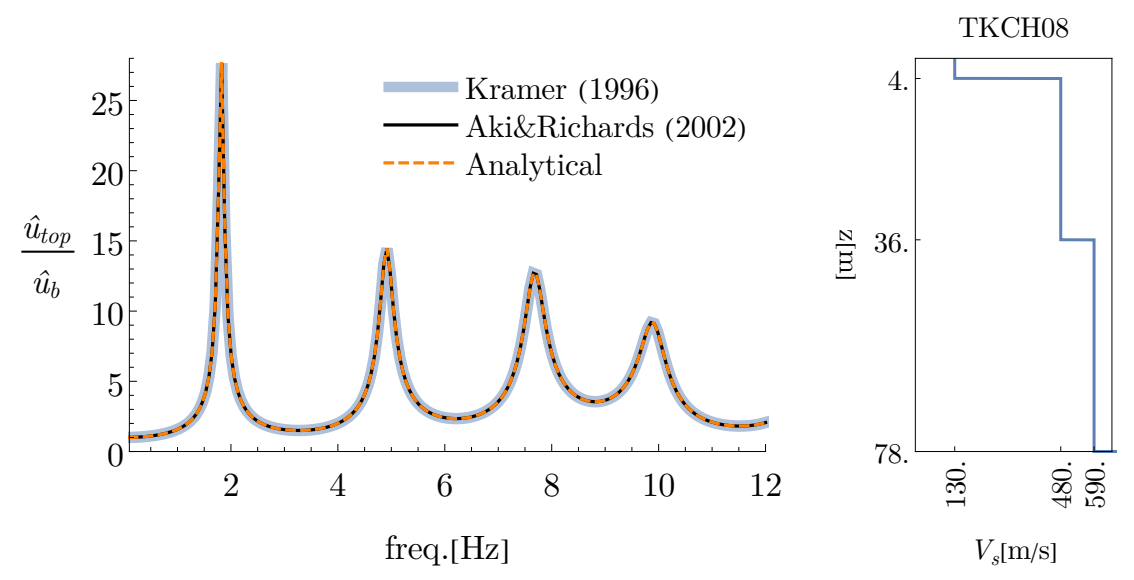

Figure 2: Results for the transfer function displacement-to-displacement between the bedrock interface and the ground surface for Kik-net station TKCH08. 

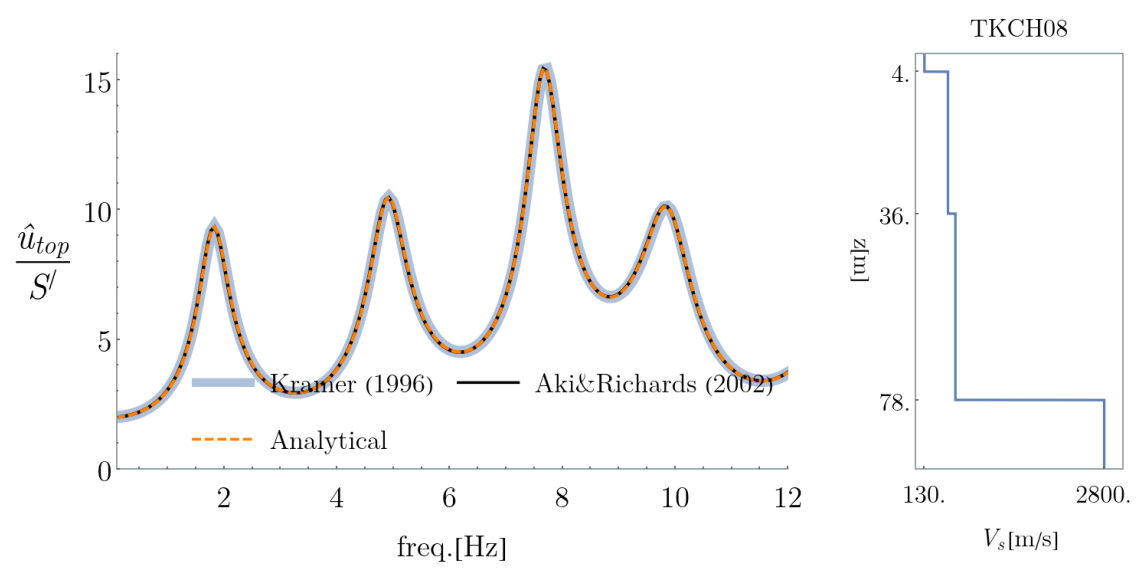

Figure 3: Results for the transfer function displacement-to-amplitude between the bedrock interface and the ground surface for Kik-net station TKCH08.
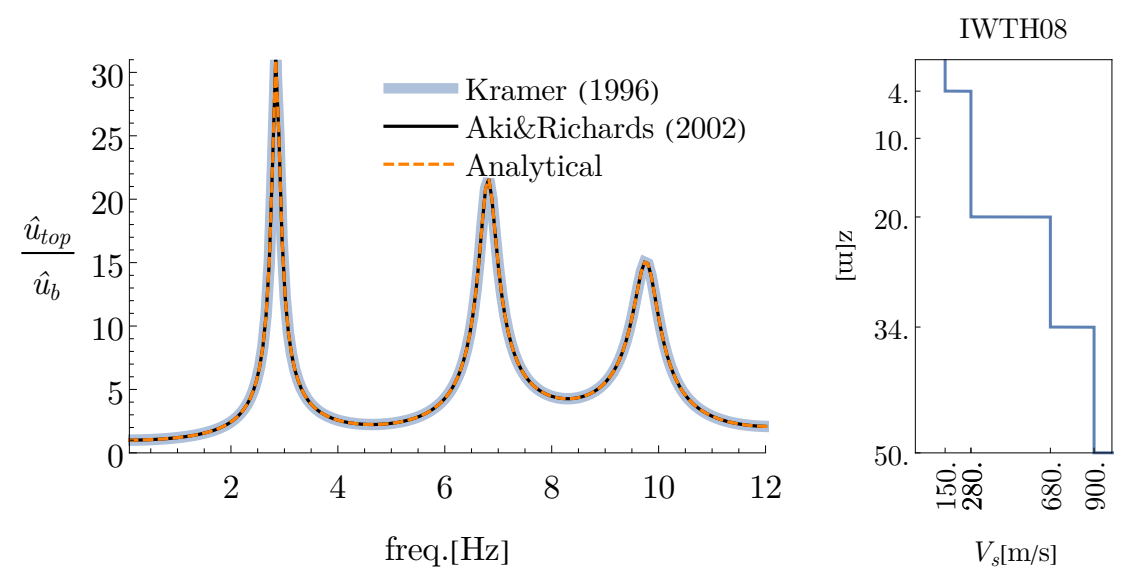

Figure 4: Results for the transfer function displacement-to-displacement between the bedrock interface and the ground surface for Kik-net station IWTH08. 

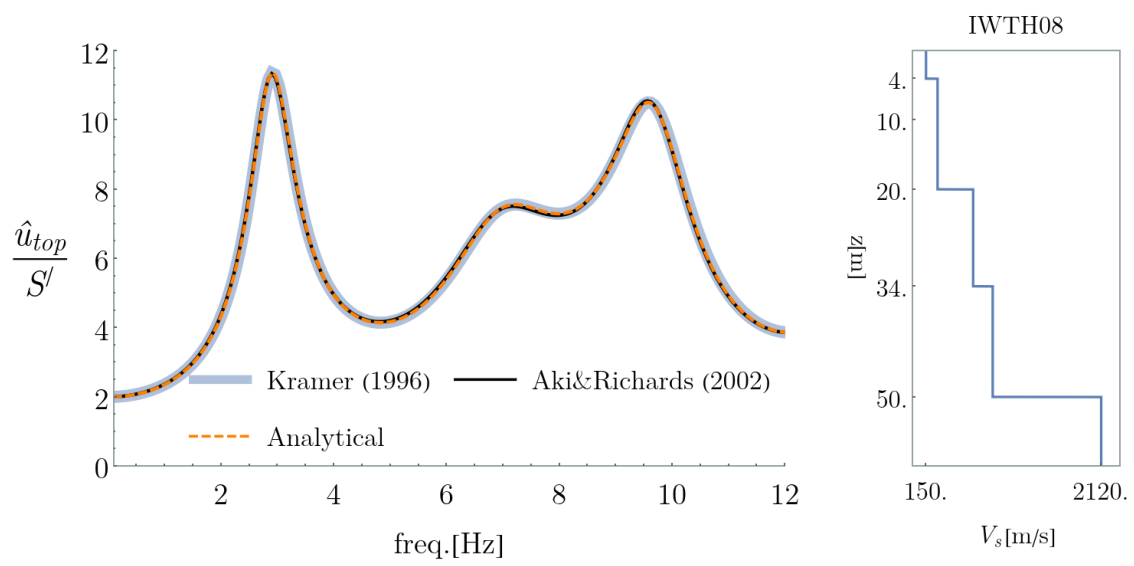

Figure 5: Results for the transfer function displacement-to-amplitude between the bedrock interface and the ground surface for Kik-net station IWTH08.

\section{Application: long-wavelength asymptotics of averaged horizontal-to-vertical spectral ratios}

The work by Kawase, Sanchez-Sesma and Matsushima [5] outlined the relation between the transfer functions and the HV spectral ratio in the context of Diffusive-Field Theory. The authors established a relation between the imaginary part of the Green's functions in a layered site, under the 1D linear-elastic propagation assumption, and the ratio of the horizontal spectral displacement amplitude and the vertical one. They validated the results of their development using both seismogram synthetics and real data, and proved both the convergence to their average result and its robustness (its ability to predict results also in scenarios that did not fit perfectly the assumptions used during the derivations, e.g., inclined wave field with mode conversion, a possibility that was not included in their analysis but it was shown that the effects of this wave inclination is smoothed out by the intrinsic averaging after superimposing many cases of different inclinations).

In summary, this work proved that transfer functions could be used to compute the average horizontal-to-vertical spectral ratios both at the surface and at some depth, thus they can serve as a tool in Inverse Analysis when it comes to identify the soil profile from ground and borehole records. We refer the reader to the original appear for more information concerning the limitations of the results in terms of number of records to average over, earthquake magnitude and Peak Ground Acceleration.

Adopting the notation in [5], we will use $\mathrm{TF}_{p}$ to refer to $\mathrm{P}$-wave transfer function (horizontal displacement) while $\mathrm{TF}_{s}$ does to S-wave transfer function. 
The following relation between the HV ratio (evaluated at the surface) and displacement-to-amplitude transfer function was found:

$$
\mathrm{rHV}=\frac{H(0 ; \omega)}{V(0 ; \omega)}=\sqrt[4]{\frac{8\left(1-\nu_{\text {half }}\right)}{\left(1-2 \nu_{\text {half }}\right)}} \frac{\left|\mathrm{TF}_{s}\right|}{\left|\mathrm{TF}_{p}\right|},
$$

where $\nu_{\text {half }}$ represents the Poisson's ratio of the half-space material.

The result eq. (4) provides the horizontal displacement, the vertical, invoking the 1D-propagation assumption, is obtained from the same expression, simply substituting $\mu$ by $\lambda+2 \mu$, to consider pressure waves.

The transfer functions that appear in [5] represent the same concepts that we have been handling in this manuscript, and hence we are in a position to offer a contribution:

It is known that the low-frequency (long-wavelength) asymptote goes to a constant value that only depends on the Poisson's ratio of the underlying half-space (in the case of it being a Poisson's solid, $\nu_{\text {half }}=0.25$, then the asymptote is $\sqrt{2 \sqrt{3}} \approx 1.86$ ), but, even though the asymptotic value can be computed, a general approximation of the ratio as it tends to that limit is not available and thus it must be obtained numerically along with the rest of the ratio for larger values of frequencies.

\subsection{Derivation of asymptotic result}

Having eq. (16) under our belt, we aim at Taylor-expanding it in the longwavelength limit (i.e., around $\omega=0$ ) to then use the expansion to achieve the long-wavelength asymptotic approximation of eq. (21). We shall provide the first term of the Taylor expansion of rHV, that is, the initial slope of the ratio, for any layering. This formula could be used later to perform a preliminary profile inversion using averaged horizontal-to-vertical ratios at different depths before resorting to numerical computations.

First, see that in the low-frequency (long-wavelength) limit we can state that $\omega h_{i} / \sqrt{\mu_{i} / \rho_{i}} \ll 1$ for any $i=1 \ldots N$. This means that all the arguments of the tangents and cosines that appear in the analytical expressions, $r_{i}$ for $i=1 \ldots N$, are very small, hence we consider the long-wavelength regime to correspond to $r_{i} \rightarrow 0$. Therefore eq. (7) yields

$$
\lim _{r_{1} \ldots r_{N} \rightarrow 0} T F_{u-u}=1,
$$

while

$$
\lim _{r_{1} \ldots r_{N} \rightarrow 0} \frac{P_{21}}{\omega \sqrt{\rho_{\text {half }} \mu_{\text {half }}}}=0
$$

so it follows that

$$
\lim _{r_{1} \ldots r_{N} \rightarrow 0} \mathrm{TF}_{p}=\lim _{r_{1} \ldots r_{N} \rightarrow 0} \mathrm{TF}_{s}=1
$$


Combining, the expression of the horizontal-vertical amplitude ratio in the long-wavelength regime is finally obtained:

$$
\mathrm{rHV} \approx \sqrt[4]{\frac{8\left(1-\nu_{\text {half }}\right)}{\left(1-2 \nu_{\text {half }}\right)}},
$$

for $\omega \ll \min _{i=1, \ldots, N}\left\{c_{s, i} / h_{i}\right\}$.

Additionally, the analytical formulae can also be used to estimate the gradient and the curvature of the ratio as a function of frequency in this low-frequency regime. To do so, we need to remove higher-order terms in the Taylor-expansion of the tangents and cosines around $r_{i}=0$. Let us split the job and begin from eq. (7):

$$
\begin{aligned}
T F_{u-u}^{-1} & =\left(\prod_{i=1}^{N} \cos \left(r_{i}\right)\right)\left(\sum_{\beta=0}^{\lfloor N / 2\rfloor} \sum_{|\mathrm{b}|=2 \beta} C_{\mathrm{b}} \tan (r)^{\mathrm{b}}\right) \\
& =\left(1-\frac{1}{2} \sum_{i=1}^{N}\left(\frac{\omega h_{i}}{\sqrt{\mu_{i} / \rho_{i}}}\right)^{2}+\mathcal{O}\left(\omega^{4}\right)\right)\left(1+\sum_{|\mathrm{b}|=2} C_{\mathrm{b}}(r)^{\mathrm{b}}+\mathcal{O}\left(\omega^{4}\right)\right) \\
& =1-\frac{1}{2} \sum_{i=1}^{N}\left(\frac{\omega h_{i}}{\sqrt{\mu_{i} / \rho_{i}}}\right)^{2}+\sum_{|\mathrm{b}|=2}(-1)^{|\mathrm{b}| / 2}(\sqrt{\rho \mu})^{\mathrm{b}^{\prime}}\left(\frac{\omega h}{\sqrt{\mu / \rho}}\right)^{\mathrm{b}}+\mathcal{O}\left(\omega^{4}\right) \\
& =1-\frac{\omega^{2}}{2} \sum_{i=1}^{N}\left(\frac{h_{i}}{\sqrt{\mu_{i} / \rho_{i}}}\right)^{2} \\
& -\omega^{2} \sum_{i=1}^{N-1} \sum_{j>i}^{N} \sqrt{\frac{\rho_{i} \mu_{i}}{\rho_{j} \mu_{j}}}\left(\frac{h_{i}}{\sqrt{\mu_{i} / \rho_{i}}}\right)\left(\frac{h_{j}}{\sqrt{\mu_{j} / \rho_{j}}}\right)+\mathcal{O}\left(\omega^{4}\right) \\
& =1-\frac{\omega^{2}}{2}\left(\sum_{i=1}^{N} t_{i}^{2}+2 \sum_{i=1}^{N-1} \sum_{j>i}^{N} \mathcal{Z}_{i, j} t_{i} t_{j}\right)+\mathcal{O}\left(\omega^{4}\right) \\
& =1-\frac{\kappa}{2} \omega^{2}+\mathcal{O}\left(\omega^{4}\right),
\end{aligned}
$$

where $t_{i}=h_{i} / \sqrt{\mu_{i} / \rho_{i}}$ represents the (shear) wave travel time in the $i$-th layer, and thus

$$
\kappa=\sum_{i=1}^{N} t_{i}^{2}+2 \sum_{i=1}^{N-1} \sum_{j>i}^{N} \mathcal{Z}_{i, j} t_{i} t_{j},
$$

represents the curvature of the transfer function in the low-frequency regime, while $\mathcal{Z}_{i, j}$ represents a matrix whose entries are function of the interface impedance 
contrasts:

$$
\mathcal{Z}_{i, j}=\prod_{k=i}^{j} Z_{k},
$$

and where, to conclude, each interface impedance contrast itself is given by

$$
Z_{k}=\frac{\sqrt{\rho_{k} \mu_{k}}}{\sqrt{\rho_{k+1} \mu_{k+1}}} .
$$

Following with the second part of eq. (6), the one that depends on $P_{21}$ :

$$
\begin{aligned}
\frac{P_{21}}{\omega \sqrt{\rho_{\text {half }} \mu_{\text {half }}}} & =\underbrace{\left(\prod_{i=1}^{N} \cos \left(r_{i}\right)\right)}_{1+\mathcal{O}\left(\omega^{2}\right)}\left(\sum_{\beta=0}^{\left\lfloor\frac{N-1}{2}\right\rfloor} \sum_{|\mathbf{b}|=1+2 \beta} \frac{S_{\mathbf{b}}}{\sqrt{\rho_{\text {half }} \mu_{\text {half }}}} \tan (r)^{\mathbf{b}}\right) \\
& =\left(1+\mathcal{O}\left(\omega^{2}\right)\right)\left(\sum_{|\mathbf{b}|=1} \frac{S_{\mathbf{b}}}{\sqrt{\rho_{h a l f} \mu_{h a l f}}}(r)^{\mathbf{b}}+\mathcal{O}\left(\omega^{3}\right)\right) \\
& =\omega \sum_{|\mathbf{b}|=1} \frac{(-1)^{(|\mathbf{b}|+1) / 2}(\sqrt{\rho \mu})^{\mathrm{g}(\mathbf{b})}}{\sqrt{\rho_{h a l f} \mu_{h a l f}}}\left(\frac{\omega h}{\sqrt{\rho \mu}}\right)^{\mathbf{b}}+\mathcal{O}\left(\omega^{3}\right) \\
& =-\omega \sum_{i=1}^{N} \sqrt{\frac{\rho_{i} \mu_{i}}{\rho_{\text {half }} \mu_{h a l f}}}\left(\frac{\omega h_{i}}{\sqrt{\rho_{i} \mu_{i}}}\right)+\mathcal{O}\left(\omega^{3}\right) \\
& =-\gamma \omega+\mathcal{O}\left(\omega^{3}\right),
\end{aligned}
$$

where

$$
\gamma=\sum_{i=1}^{N} \mathcal{Z}_{i, N+1} t_{i}
$$

Combining the two previous results into eq. (6) yields, finally,

$$
2(\mathrm{TF})^{-1}=1-\mathrm{i} \gamma \omega-\frac{\kappa}{2} \omega^{2}+\mathcal{O}\left(\omega^{3}\right)
$$

This result is verified in Figures 6 and 7 (the same profiles considered in Section 3 are used again) by plotting the real and imaginary part of eq. (32) alongside the real and imaginary part of eq. (16). As it was expected, the accuracy of the long-wavelength approximation deteriorates as the higher-order terms become significant for higher frequencies. The real part, associated to the quadratic term, is well captured in both cases.

Note in Figures 4 and 5 that the first resonance of the site "IWTH08" happens around $2 \mathrm{~Hz}$, while in Figure 7 the approximation departs from the exact values as it approaches precisely that value, hence we can say that the approximation is able of reflecting the behavior prior to fundamental resonance. Likewise, the first resonance peak is located around $2 \mathrm{~Hz}$ in the case of "TKCH08", 


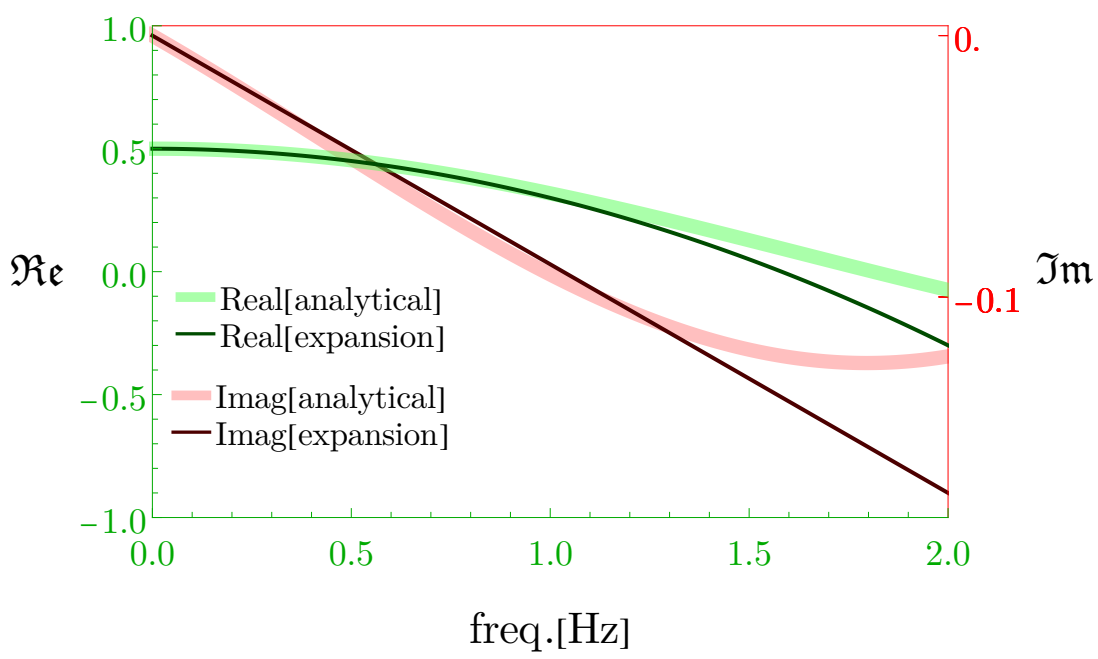

Figure 6: Real and imaginary parts of displacement-amplitude transfer function in eq. (16) (thicker lines) and the corresponding long-wavelength approximation eq. (32) for the site TKCH08. Notice the two different vertical axes.

Figures 2 and 3, and gazing at Figure 6 we realize that the same behavior is observed: the approximation works well all the way up to interval close to the value of the first resonance frequency.

Using the latest expression we can approximate rHV in the long-wavelength regime. Let us work with eq. (21) squared, to easy the derivation, thus

$\left.\mathrm{rHV}\right|_{l w} ^{2}=\sqrt{\frac{8\left(1-\nu_{h a l f}\right)}{\left(1-2 \nu_{h a l f}\right)}} \frac{\left|1-\frac{\kappa_{p}}{2} \omega^{2}-\mathrm{i} \gamma_{p} \omega+\mathcal{O}\left(\omega^{3}\right)\right|^{2}}{\left|1-\frac{\kappa_{s}}{2} \omega^{2}-\mathrm{i} \gamma_{s} \omega+\mathcal{O}\left(\omega^{3}\right)\right|^{2}}$,

compute the absolute value of the complex numbers by multiplying by the conjugate

$$
=\sqrt{\frac{8\left(1-\nu_{\text {half }}\right)}{\left(1-2 \nu_{\text {half }}\right)}} \frac{\left(1-\frac{\kappa_{p}}{2} \omega^{2}\right)^{2}+\gamma_{p}^{2} \omega^{2}+\mathcal{O}\left(\omega^{3}\right)}{\left(1-\frac{\kappa_{s}}{2} \omega^{2}\right)^{2}+\gamma_{s}^{2} \omega^{2}+\mathcal{O}\left(\omega^{3}\right)},
$$

expanding the square in the first addends,

$$
\begin{aligned}
& =\sqrt{\frac{8\left(1-\nu_{\text {half }}\right)}{\left(1-2 \nu_{\text {half }}\right)}} \frac{\left(1-\kappa_{p} \omega^{2}+\frac{\kappa_{p}^{2}}{4} \omega^{4}\right)+\gamma_{p}^{2} \omega^{2}+\mathcal{O}\left(\omega^{3}\right)}{\left(1-\kappa_{s} \omega^{2}+\frac{\kappa_{s}^{2}}{4} \omega^{4}\right)+\gamma_{s}^{2} \omega^{2}+\mathcal{O}\left(\omega^{3}\right)}, \\
& =\sqrt{\frac{8\left(1-\nu_{\text {half }}\right)}{\left(1-2 \nu_{\text {half }}\right)}} \frac{1+\left(\gamma_{p}^{2}-\kappa_{p}\right) \omega^{2}+\mathcal{O}\left(\omega^{3}\right)}{1+\left(\gamma_{s}^{2}-\kappa_{s}\right) \omega^{2}+\mathcal{O}\left(\omega^{3}\right)}
\end{aligned}
$$




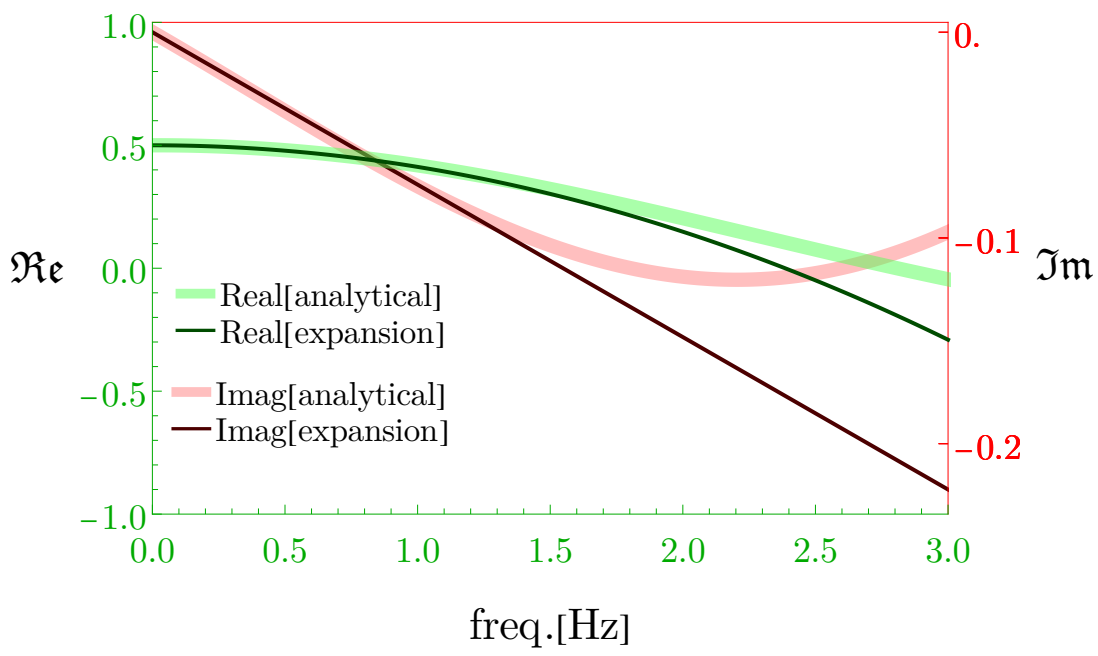

Figure 7: Real and imaginary parts of displacement-amplitude transfer function in eq. (16) (thicker lines) and the corresponding long-wavelength approximation eq. (32) for the site IWTH08. Notice the two different vertical axes. 
Taylor-expanding the denominator around $\omega=0$,

$$
\begin{aligned}
& =\sqrt{\frac{8\left(1-\nu_{\text {half }}\right)}{\left(1-2 \nu_{\text {half }}\right)}}\left[1+\left(\gamma_{p}^{2}-\kappa_{p}\right) \omega^{2}+\mathcal{O}\left(\omega^{3}\right)\right]\left[1-\left(\gamma_{s}^{2}-\kappa_{s}\right) \omega^{2}+\mathcal{O}\left(\omega^{3}\right)\right] \\
& =\sqrt{\frac{8\left(1-\nu_{\text {half }}\right)}{\left(1-2 \nu_{\text {half }}\right)}}\left\{1+\left[\left(\gamma_{p}^{2}-\gamma_{s}^{2}\right)+\left(\kappa_{s}-\kappa_{p}\right)\right] \omega^{2}+\mathcal{O}\left(\omega^{3}\right)\right\}
\end{aligned}
$$

To conclude, remove higher-order terms, square-root the prior expression and approximate it assuming the second addend is smaller than 1 to obtain:

$$
\left.\mathrm{rHV}\right|_{l w}=\sqrt[4]{\frac{8\left(1-\nu_{h a l f}\right)}{\left(1-2 \nu_{h a l f}\right)}}\left\{1+\frac{\left[\left(\gamma_{p}^{2}-\gamma_{s}^{2}\right)+\left(\kappa_{s}-\kappa_{p}\right)\right]}{2} \omega^{2}\right\} .
$$

$\gamma_{p}$ and $\gamma_{s}$ are the slopes of the transfer functions for P-waves and S-waves, the only difference between them that one has to use $\lambda+2 \mu$ instead of $\mu$ when it comes to compute layer travel times and impedance ratios, and, likewise, $\kappa_{p}$ and $\kappa_{s}$ are the corresponding curvatures of the transfer functions.

The previous result indicates that the frequency plot of horizontal-to-vertical ratio should take off with zero slope but with a certain curvature. The latter asseveration appears consistent with the results in [5] (see their images where they present HV ratios, in particular, Figures 6, 7, 8. 10, 11 and 13 in their paper).

The formula reveals that the curvature at the origin of the averaged horizontalto-vertical ratio depends on the compressibility of the underlying halfspace through the coefficients obtained from equipartition of energy (first factor where $\nu_{\text {half }}$ appears), and through the slope and curvature at the origin of the transfer functions, which depend also on the mechanical properties of the layering up tp the half-space (via the inter-layer impedances that appear in the expressions) and on the thickness of each layer (via the travel times, also present within eqs. (27) and (31)).

\section{Final Remarks}

The general closed-form expressions for the customary transfer functions in a 1D layered site, for any number of layers, have been introduced in this text, eq. (7) and eq. (16). It has been shown that they adopt a compact, manageable form. This fact may pave the way to tackle questions concerning seismic response of layered sites using an analytical approach.

These expressions allow to obtain useful information right away; in particular, we have provided the low-frequency slope and curvature of the transfer functions, and used this result to particularize the expression of the ratio of horizontal-to-vertical surface average spectral displacements, as presented in [5]. Using the closed-form expression and their long-wavelength expansions, an approximate formula of the ratio has been retrieved, see eq. (34). The formula 
is valid as long as wavelengths in all layers are greater than their thickness, so it could potentially capture the behavior up to the first resonance peak. Direct comparison of eq. (34) to numerical computations of the ratios from both synthetics and real earthquake records, in the spirit of the results presented in [5], remains an open task; nevertheless, we are confident that such comparison would be satisfactory, based on the verification exercise displayed in Figures 6 and 7. Once this task is finished, eq. (34) could be used to guide the inversion of site profiles from seismic records [16].

These analytical expressions can be further explored in order to assess, for instance:

- The influence of the presence of velocity reversals in a site [11]. The prowess of an analytical treatment of the problem has been already demonstrated, see [17]

- The definition of equivalent-homogeneous properties of inhomogeneous sites $[18,19,20,21]$.

The 1D-propagation of decoupled waves is not the only wave phenomenon amenable to be treated via the transfer matrices: surface waves (both Love's and Rayleigh's) and inclined bulk waves (both SH waves and P-SV waves) also lend themselves to this treatment (details on this can be found in [1]). This means that the understanding of the aforementioned problems could also benefit from the knowledge of the exact form of the global propagator. See the conclusions in [8] for more future work in this direction.

\section{Acknowledgements}

The Authors are thankful to Prof. Elnaz E. Seylabi (University of Nevada, Reno) for suggesting the application.

\section{Supplementary Material}

A Mathematica notebook [15] containing the computations leading to results (including figures) shown in the text can be found in the repository named layered_TFs in the first author's GitHub page github.com/jgarciasuarez.

\section{References}

[1] Keiiti Aki and Paul G Richards. Quantitative seismology. University Science Books, 2002.

[2] Steven L Kramer. Geotechnical earthquake engineering. in Prentice-Hall international series in civil engineering and engineering mechanics. Prentice Hall Inc., Englewood Cliffs, New Jersey, 1996. 
[3] Freeman Gilbert and George E Backus. Propagator matrices in elastic wave and vibration problems. Geophysics, 31(2):326-332, 1966.

[4] Ari Ben-Menahem and Sarva Jit Singh. Seismic waves and sources. Springer Science \& Business Media, 2012.

[5] Hiroshi Kawase, Francisco J Sánchez-Sesma, and Shinichi Matsushima. The optimal use of horizontal-to-vertical spectral ratios of earthquake motions for velocity inversions based on diffuse-field theory for plane waves. Bulletin of the Seismological Society of America, 101(5):2001-2014, 2011.

[6] Lorenzo Morini, Zafar Gokay Tetik, Gal Shmuel, and Massimiliano Gei. On the universality of the frequency spectrum and band-gap optimization of quasicrystalline-generated structured rods. Philosophical Transactions of the Royal Society A, 378(2162):20190240, 2020.

[7] Gal Shmuel and Ram Band. Universality of the frequency spectrum of laminates. Journal of the Mechanics and Physics of Solids, 92:127-136, 2016.

[8] Joaquin Garcia-Suarez. Trace spectrum of 1d transfer matrices for wave propagation in layered media. engrxiv.org/ygt8z, Jun 2021.

[9] John Lekner. Light in periodically stratified media. JOSA A, 11(11):28922899, 1994.

[10] Mingrong Shen and Wenwu Cao. Acoustic bandgap formation in a periodic structure with multilayer unit cells. Journal of Physics D: Applied Physics, 33(10):1150, 2000.

[11] Gony Yagoda-Biran, Bibi Kerpel, and Ronnie Kamai. Never fear velocity reversalsshort note. Bulletin of the Seismological Society of America, 107(4):1969-1974, 2017.

[12] Gregory A Madera. Fundamental period and amplification of peak acceleration in layered systems, massachusetts research report $\mathrm{r}$ 70-37. MIT Press, 1970.

[13] Yoshimitsu Okada, Keiji Kasahara, Sadaki Hori, Kazushige Obara, Shoji Sekiguchi, Hiroyuki Fujiwara, and Akira Yamamoto. Recent progress of seismic observation networks in japan - hi-net, f-net, k-net and kik-netEarth, Planets and Space, 56(8):xv-xxviii, 2004.

[14] James Kaklamanos, Laurie G Baise, Eric M Thompson, and Luis Dorfmann. Comparison of $1 \mathrm{~d}$ linear, equivalent-linear, and nonlinear site response models at six kik-net validation sites. Soil Dynamics and Earthquake Engineering, 69:207-219, 2015.

[15] Stephen Wolfram. The mathematica book, volume 4. Cambridge University Press Cambridge, 2000. 
[16] Elnaz Seylabi, Andrew M. Stuart, and Domniki Asimaki. Site Characterization at Downhole Arrays by Joint Inversion of Dispersion Data and Acceleration Time Series. Bulletin of the Seismological Society of America, 110(3):1323-1337, June 2020.

[17] Joaquin Garcia-Suarez. Utilizing analytical transfer functions to gauge the effect of velocity reversals. engrxiv.org/vt6jq, Jun 2021.

[18] Christos Vrettos. Dynamic response of soil deposits to vertical sh waves for different rigidity depth-gradients. Soil Dynamics and Earthquake Engineering, 47:41-50, 2013.

[19] Joaquin Garcia-Suarez, E Seylabi, and Domniki Asimaki. Seismic harmonic response of inhomogeneous soil: scaling analysis. Géotechnique, 2021.

[20] Joaquin Garcia-Suarez, Domniki Asimaki, and Elnaz E Seylabi. Geometrical optics applied to 1d site response of inhomogeneous soil deposits. engrxiv.org/db7jv, Feb 2020.

[21] Joaquin Garcia-Suarez and Domniki Asimaki. On the fundamental resonant mode of inhomogeneous soil deposits. Soil Dynamics and Earthquake Engineering, 135:106190, 2020.

\section{A Brief review of multi-index notation}

A binary multi-index $\mathrm{b} \in(\{0,1\})^{N}$ is an $N$-tuple of numbers, in this case being either 0 or 1 . Its "degree" is equal to $|b|=b_{1}+\ldots+b_{N}$, thus, in this case, the degree is equal to the number of non-zero elements.

When applied to a variable, call it $x \in \mathbb{R}^{N}$, it yields

$$
x^{\mathrm{b}}=x_{1}^{\mathrm{b}_{1}} \ldots x_{N}^{\mathrm{b}_{N}} .
$$

Note that a certain polynomial of degree $N, p(x) \in \mathbb{P}_{N}(\mathbb{R})$, can be written as

$$
p(x)=\sum_{\beta=0}^{\lfloor N / 2\rfloor} \sum_{|\mathrm{b}|=2 \beta} C_{\mathrm{b}} x^{\mathrm{b}}
$$

hence, for instance, if $N=3$ :

$$
\begin{aligned}
& \text { for }|\mathbf{b}|=0:\left\{x_{1}^{0} x_{2}^{0} x_{3}^{0}\right\}=\{1\}, \\
& \text { for }|\mathbf{b}|=2:\left\{x_{1}^{1} x_{2}^{1} x_{3}^{0}, x_{1}^{1} x_{2}^{0} x_{3}^{1}, x_{1}^{0} x_{2}^{1} x_{3}^{1}\right\}=\left\{x_{1} x_{2}, x_{1} x_{3}, x_{2} x_{3}\right\},
\end{aligned}
$$

hence, it defines the following polynomial:

$$
p(x)=\sum_{\beta=0}^{1} \sum_{|\mathbf{b}|=2 \beta} C_{\mathrm{b}} x^{\mathrm{b}}=C_{(0,0,0)}+C_{(1,1,0)} x_{1} x_{2}+C_{(1,0,1)} x_{1} x_{3}+C_{(0,1,1)} x_{2} x_{3} .
$$


Yet another example, if $N=4$ :

for $|\mathrm{b}|=0:\left\{x_{1}^{0} x_{2}^{0} x_{3}^{0} x_{4}^{0}\right\}=\{1\}$,

for $|\mathrm{b}|=2:\left\{x_{1}^{1} x_{2}^{1} x_{3}^{0} x_{4}^{0}, x_{1}^{1} x_{2}^{0} x_{3}^{1} x_{4}^{0}, x_{1}^{1} x_{2}^{0} x_{3}^{0} x_{4}^{1}, x_{1}^{0} x_{2}^{1} x_{3}^{1} x_{4}^{0}, x_{1}^{0} x_{2}^{1} x_{3}^{0} x_{4}^{1}, x_{1}^{0} x_{2}^{0} x_{3}^{1} x_{4}^{1}\right\}$

$$
=\left\{x_{1} x_{2}, x_{1} x_{3}, x_{1} x_{4}, x_{2} x_{3}, x_{2} x_{4}, x_{3} x_{4}\right\},
$$

for $|\mathrm{b}|=4:\left\{x_{1}^{1} x_{2}^{1} x_{3}^{1} x_{4}^{1}\right\}=\left\{x_{1} x_{2} x_{3} x_{4}\right\}$, 


\section{A.1 Extra transfer function plots}
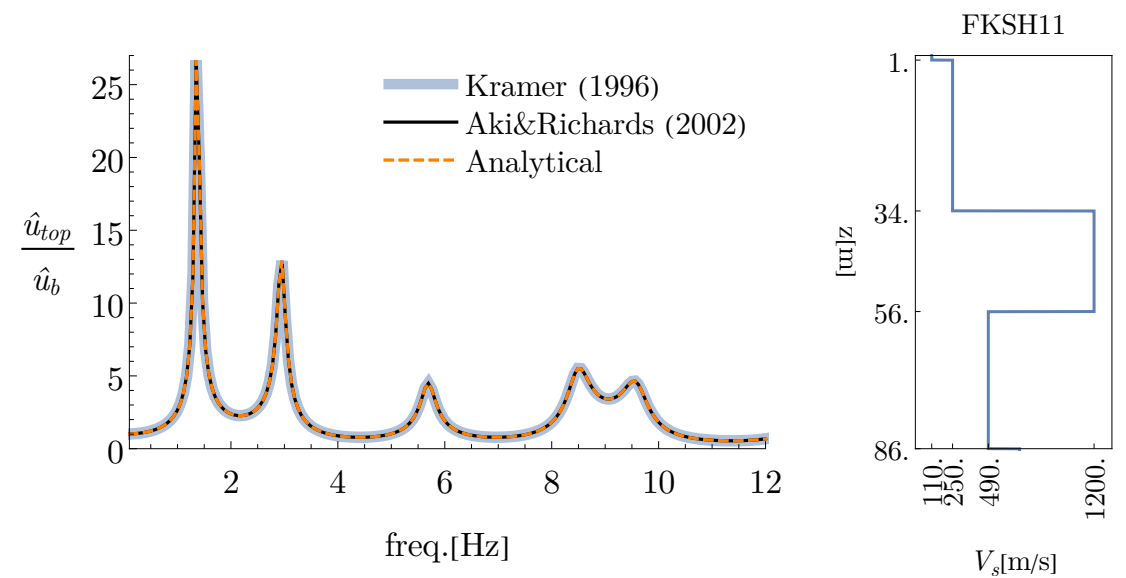

Figure 8: Results for the transfer function displacement-to-displacement between the bedrock interface and the ground surface for Kik-net station FKSH11.
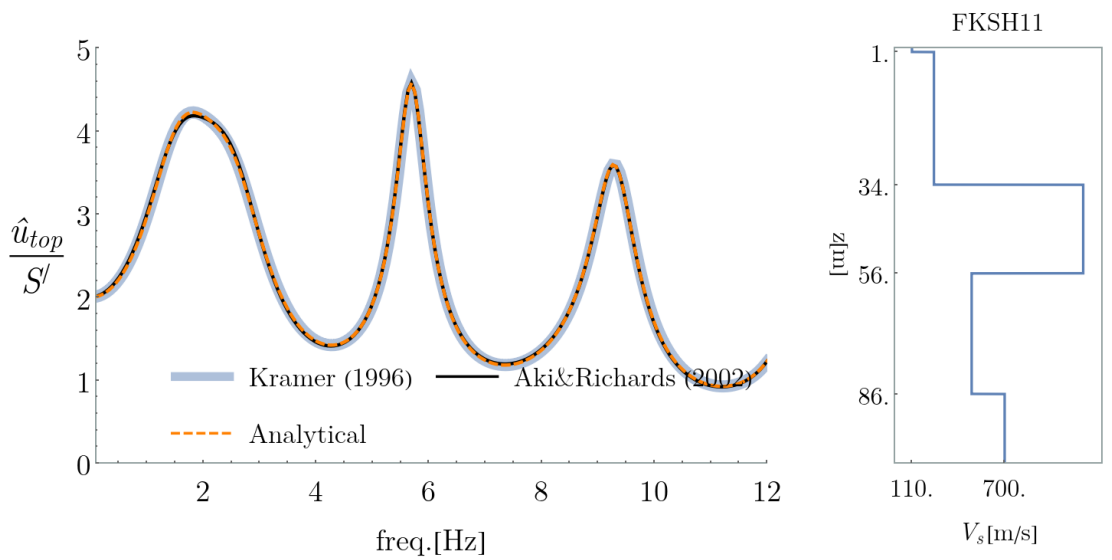

Figure 9: Results for the transfer function displacement-to-amplitude between the bedrock interface and the ground surface for Kik-net station FKSH11. 
FKSH14
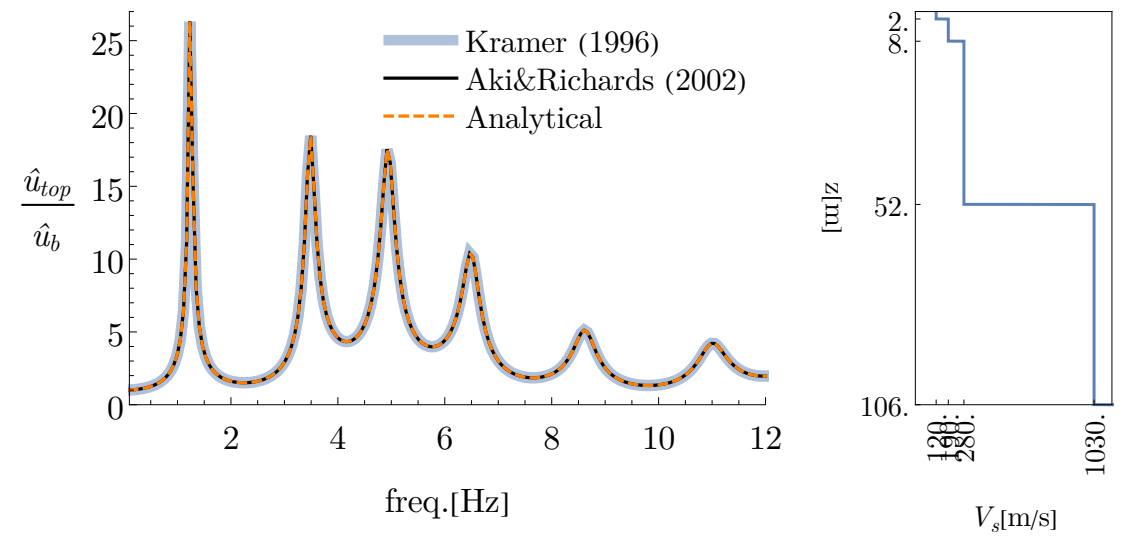

Figure 10: Results for the transfer function displacement-to-displacement between the bedrock interface and the ground surface for Kik-net station FKSH14.
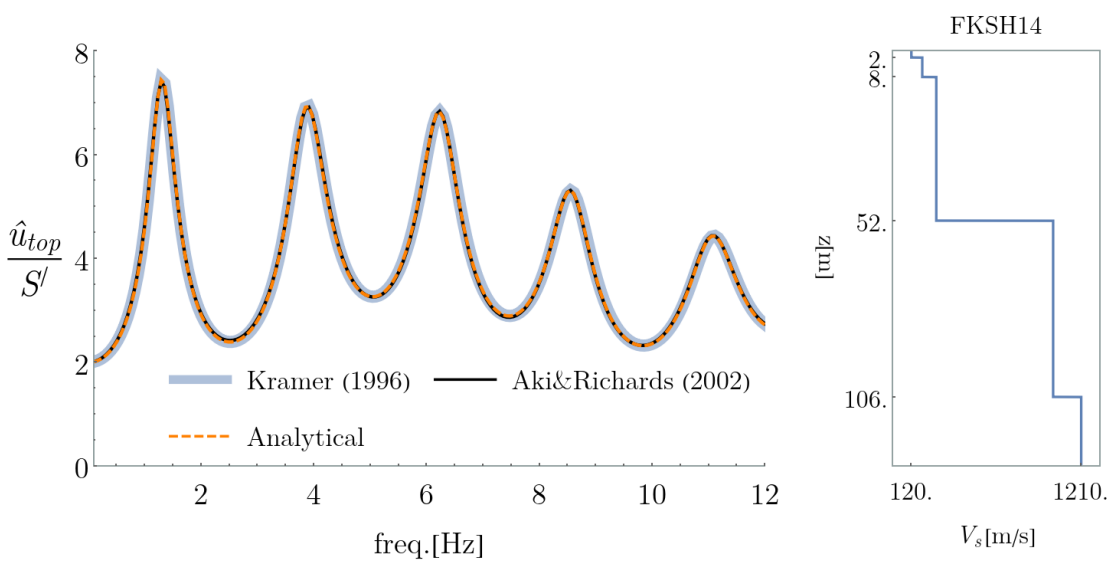

Figure 11: Results for the transfer function displacement-to-amplitude between the bedrock interface and the ground surface for Kik-net station FKSH14. 

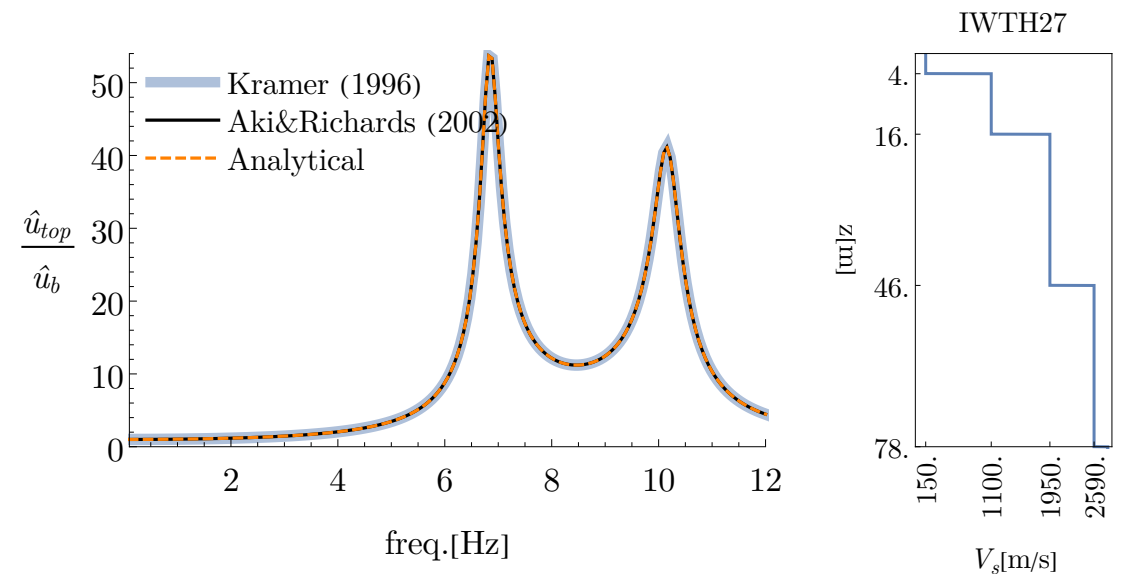

$V_{s}[\mathrm{~m} / \mathrm{s}]$

Figure 12: Results for the transfer function displacement-to-displacement between the bedrock interface and the ground surface for Kik-net station IWTH27.
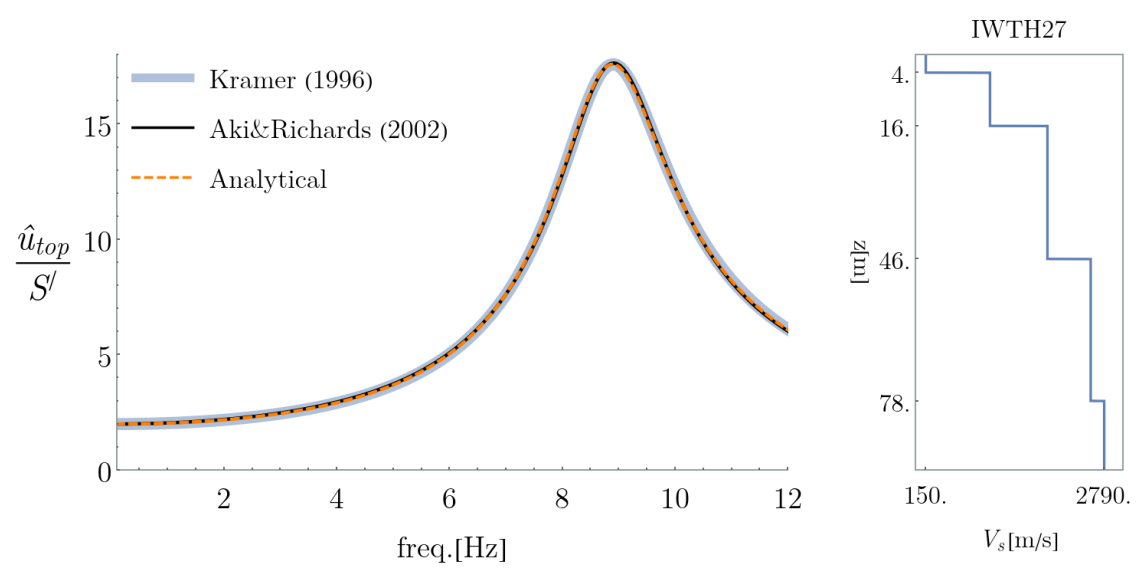

Figure 13: Results for the transfer function displacement-to-amplitude between the bedrock interface and the ground surface for Kik-net station IWTH27. 

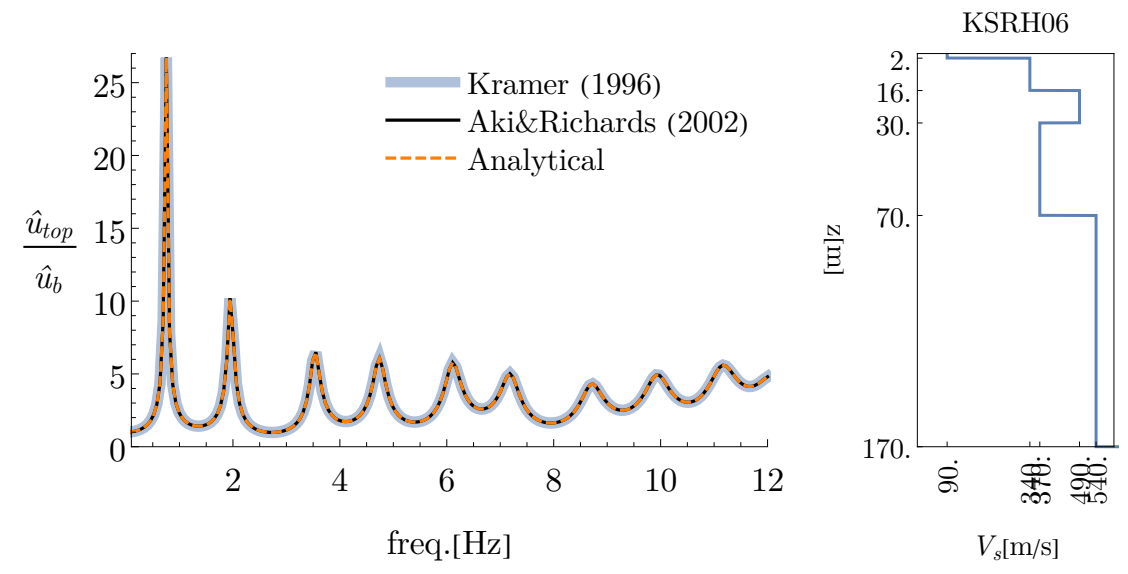

Figure 14: Results for the transfer function displacement-to-displacement between the bedrock interface and the ground surface for Kik-net station KSRH06.
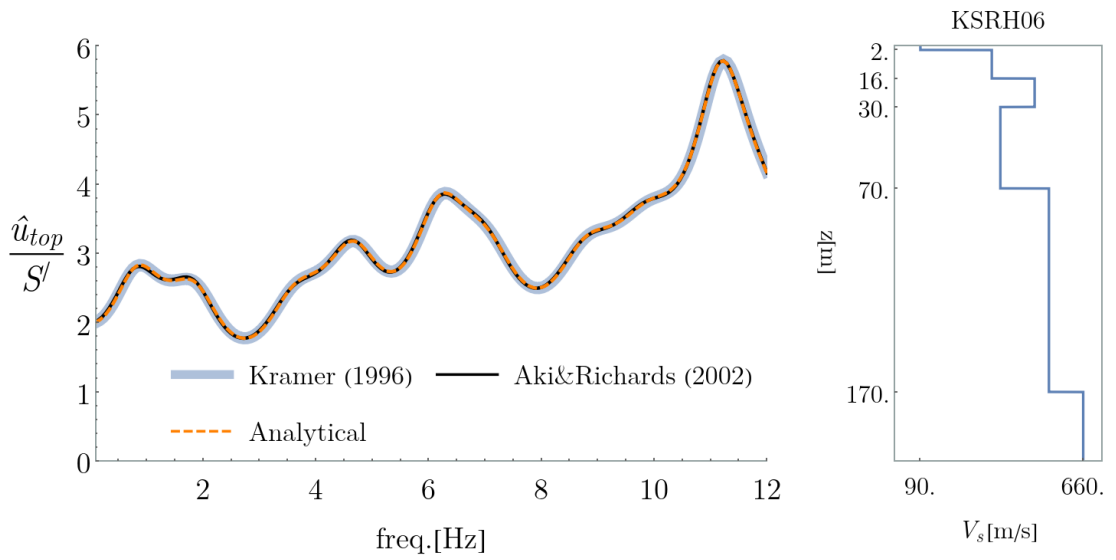

Figure 15: Results for the transfer function displacement-to-amplitude between the bedrock interface and the ground surface for Kik-net station KSRH06. 

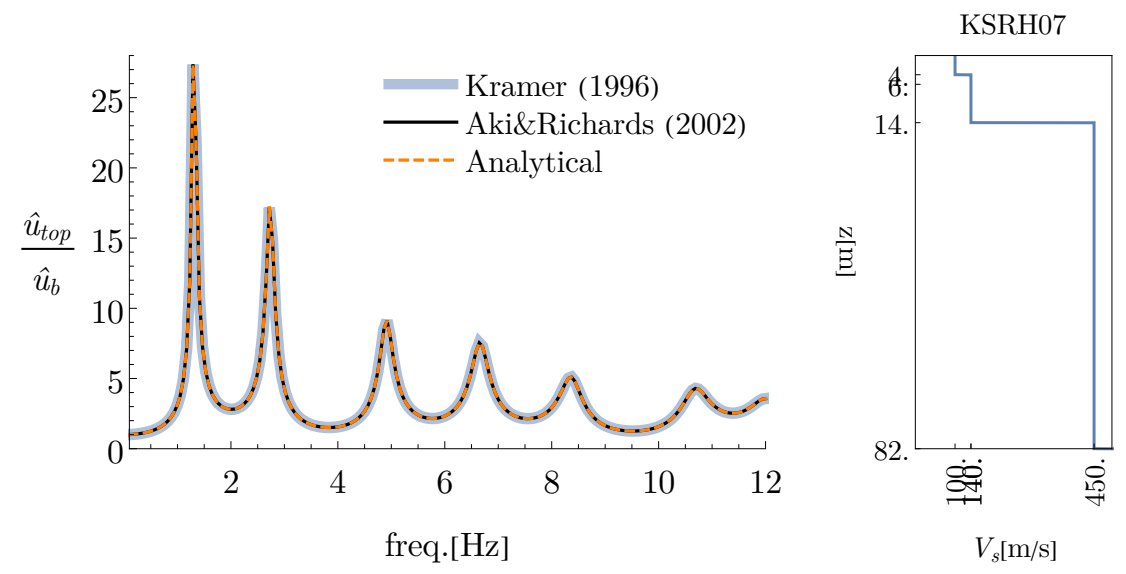

Figure 16: Results for the transfer function displacement-to-displacement between the bedrock interface and the ground surface for Kik-net station KSRH07.
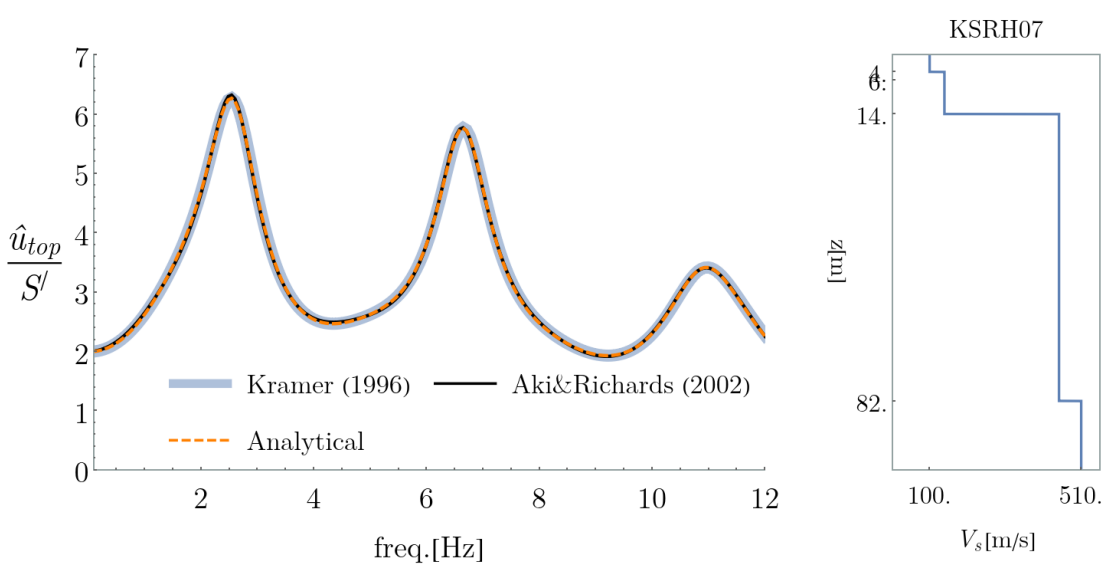

Figure 17: Results for the transfer function displacement-to-amplitude between the bedrock interface and the ground surface for Kik-net station KSRH07. 

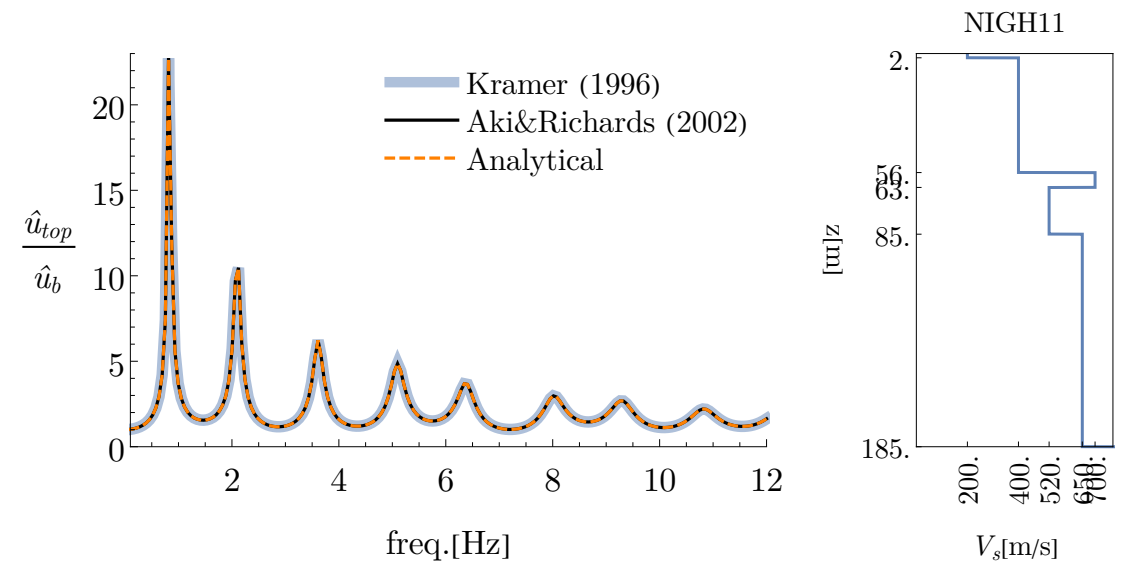

Figure 18: Results for the transfer function displacement-to-displacement between the bedrock interface and the ground surface for Kik-net station NIGH11.
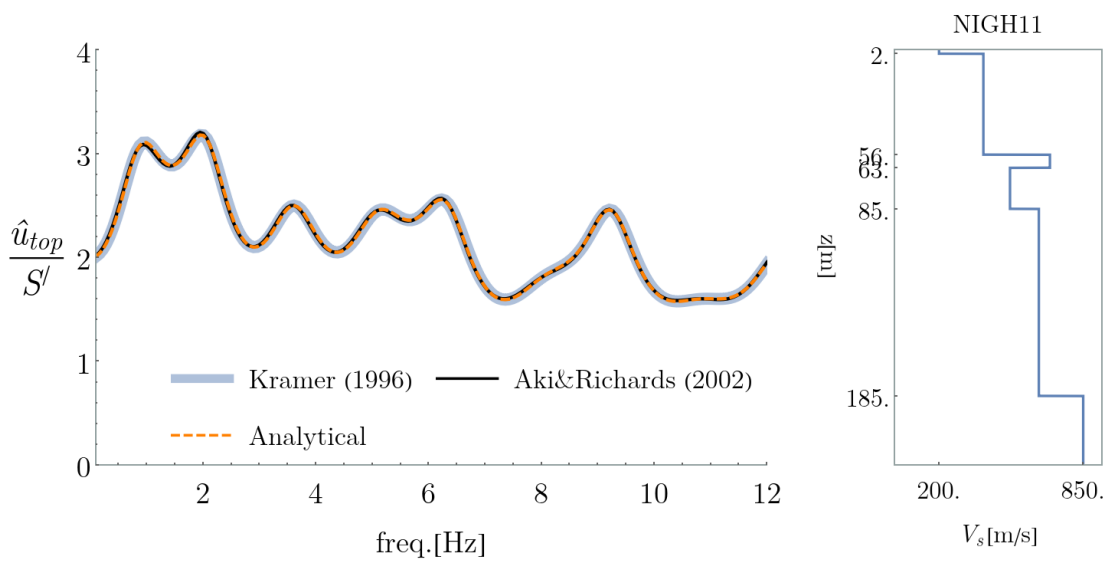

Figure 19: Results for the transfer function displacement-to-amplitude between the bedrock interface and the ground surface for Kik-net station NIGH11. 

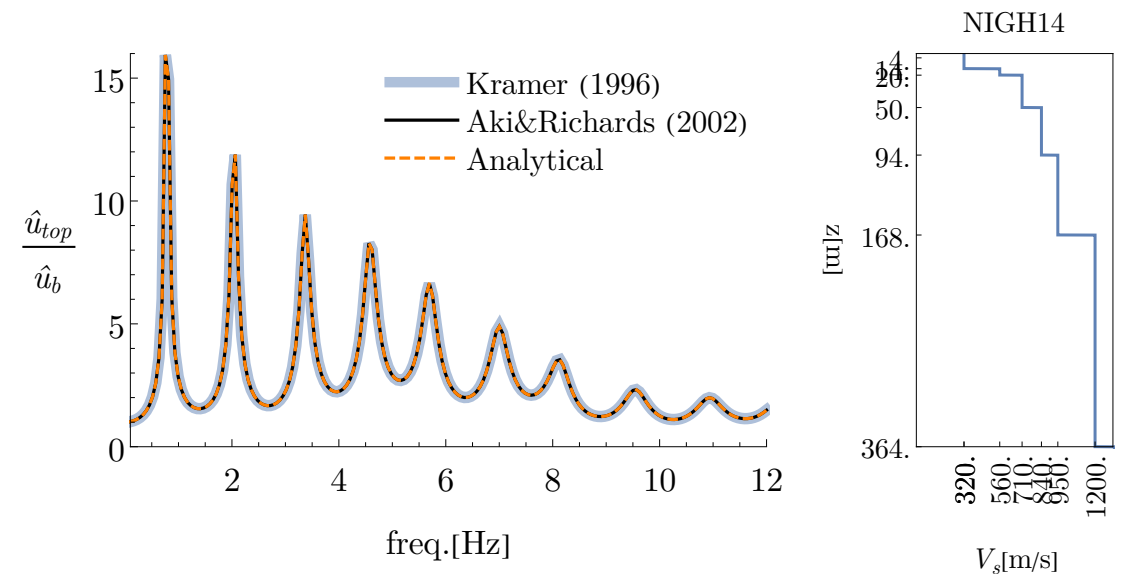

Figure 20: Results for the transfer function displacement-to-displacement between the bedrock interface and the ground surface for Kik-net station NIGH14.
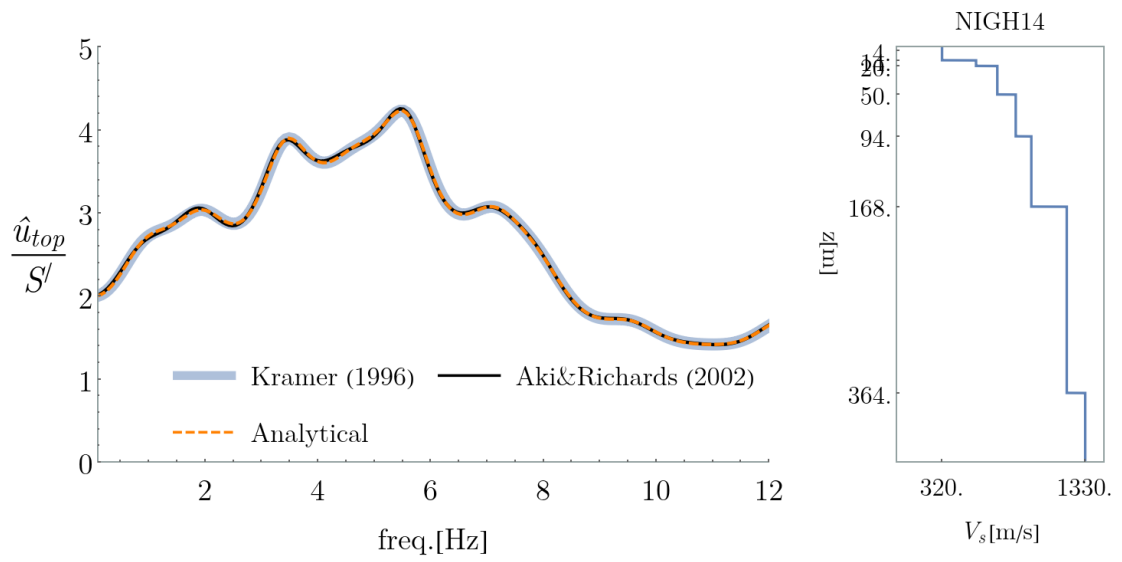

Figure 21: Results for the transfer function displacement-to-amplitude between the bedrock interface and the ground surface for Kik-net station NIGH14. 

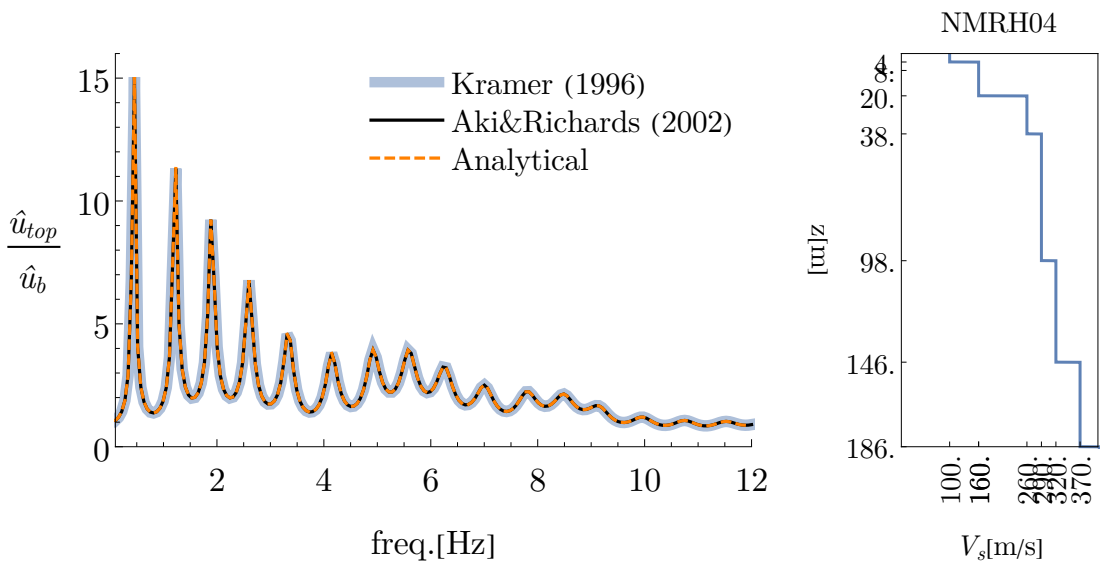

Figure 22: Results for the transfer function displacement-to-displacement between the bedrock interface and the ground surface for Kik-net station NMRH04.
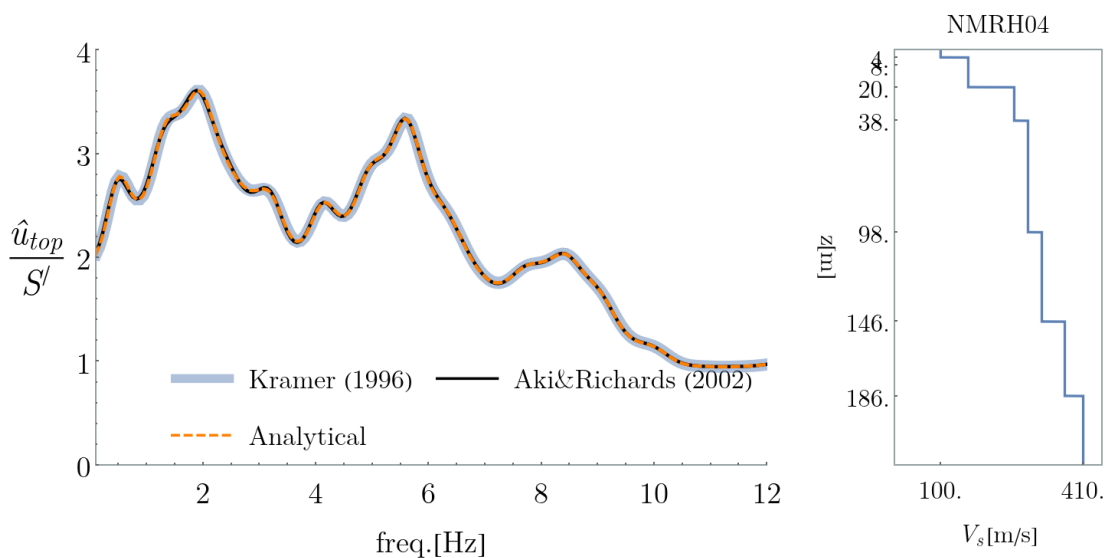

Figure 23: Results for the transfer function displacement-to-amplitude between the bedrock interface and the ground surface for Kik-net station NMRH04. 